\title{
Thèses de doctorat et mémoires de licence en géographie 2004-2006 / Doctoraats- en licentieverhandelingen geografie 2004-2006
}

\section{(2) OpenEdition}

\section{Journals}

Édition électronique

URL : http://journals.openedition.org/belgeo/11764

DOI : $10.4000 /$ belgeo.11764

ISSN : 2294-9135

Éditeur :

National Committee of Geography of Belgium, Société Royale Belge de Géographie

Édition imprimée

Date de publication : 31 décembre 2006

Pagination : 473-492

ISSN : $1377-2368$

Référence électronique

«Thèses de doctorat et mémoires de licence en géographie 2004-2006 / Doctoraats- en licentieverhandelingen geografie 2004-2006 », Belgeo [En ligne], 4 | 2006, mis en ligne le 31 octobre 2013, consulté le 01 mai 2019. URL : http://journals.openedition.org/belgeo/11764; DOI : 10.4000/ belgeo.11764

Ce document a été généré automatiquement le 1 mai 2019.

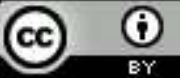

Belgeo est mis à disposition selon les termes de la licence Creative Commons Attribution 4.0 International. 


\section{Thèses de doctorat et mémoires de} licence en géographie 2004-2006 / Doctoraats- en licentieverhandelingen geografie 2004-2006

\section{NOTE DE L'ÉDITEUR}

Cette liste fait suite à celle publiée dans Belgeo, 2003, 2, pp. 195-222.

Deze lijst is een vervolg van deze gepubliceerd in Belgeo, 2003, 2, pp. 195-222.

\section{Thèses de doctorat - Doctoraatsverhandelingen}

\section{- Katholieke Universiteit Leuven}

2004

2 VAN OOST Kristof: Spatial modeling of soil redistribution processes in agricultural landscapes.

TEMMERMAN Stijn: Sedimentation on tidal marshes in the Scheldt estuary: a field and numerical modelling study.

GIMENEZ Rafael: The interaction between rill hydraulics, rill geometry and sediment detachment: an experimental approach.

8 VANWALLEGHEM Tom: Environmental change and geomorphic response in old forests of the Loess Belt: the Meerdaal Forest Case-study. 

Automatic Classification (SOOAC) Method and Application in Land Use and Land Cover Change Detection Using Remote Sensing in Central Zimbabwe.

\section{- Université Catholique de Louvain}

GYSSELS Gwen: Impact of small grain roots on soil erosion by concentrated flow and implications for soil conservation.

2006

BRYON Jeroen : De dialectische relatie tussen stadsbewoners en de toeristisch-stedelijke ruimte. Case studie Brugge.

RUYSSCHAERT Greet: Spatial and temporal variability of soil losses due to crop harvesting.

VAN DEN EECKHAUT Miet: Spatial and temporal patterns of landslides in hilly regions.

HAREGWYN Nigussie: Reservoir sedimentation in the north Ethiopian Highlands: assessment and modelling of controlling factors and impacts.

\section{- Rijksuniversiteit Gent}

2004

VAN DE WEGHE Nico: Representing and Reasoning about Moving Objects: A Qualitative Approach.

2005

DERUYTER Greta : De Atlas der Buurtwegen als archiefbron en basis voor de uitbouw van een netwerk van trage wegen in het Vlaams en het Brussels Hoofdstedelijk Gewest. aroc. Une approche pluridisciplinaire basée sur la télédétection guidée par les observations de terrain et l'intégration dans un Système d'Information Géographique.

Oriental (Maroc) : étude explorative du phénomène urbain et industriel.

VAN DE WEGHE Nico: Representing and Reasoning about Moving Objects: A Qualitative Approach.

VAN NUFFEL Nathalie: Regionalisering van de woonmarkt. Een onderzoek naar de ruimtelijke structurering van het nederzettingspatroon in Noord-België, 1990/91 1995/96.

\section{6}

DERUDDER Ben: Conceptuele evaluatie van het onderzoek over een mondiaal stedennetwerk.

2004

BAKKER M.: The Effect of Soil Erosion on Crop Productivity and Land Use Change.

COCU N.: Analysing the effect of environment and environmental change on the spatial distribution and dynamics of the aphid species, Myzus persicae (Sulzer), in Europe. 
FLAHAUT B.: Towards a sustainable road safety in Belgium. Localisation of spatial concentrations of road accidents and explanatory modelling.

ROMANOWICZ A.: The Impacts of environmental Change on Water Resources. A Case Study in the Dyle Catchment (Belgium) in support of the implementation of the Water Framework Directive.

\section{5}

CARUSO G.: Integrating urban economics and cellular automata to model periurbanisation. Spatial dynamics of residential choice in the presence of neighbourhood externalities.

VANWAMBEKE S.: Impacts of land-use change on mosquito-borne diseases in Northern Thailand.

\section{6}

DENDONCKER N.: Statistical Downscaling of Aggregate Land Use Data: Methods and Applications.

DUJARDIN C.:The Urban Agglomerations of Lyon and Brussels.

LETERME B.: Assessing pesticide leaching at the regional scale: a case study for atrazine in the Dyle catchment.

SCHMIT C.: Analysing spatial Patterns and Representations of agricultural Land Use Data.

SCOUVART M. : Une analyse quali-quantitative comparée des causes de la déforestation en Amazonie brésilienne.

\section{- Université de Liège}

\section{4}

PARTOUNE Christine: Un modèle pédagogique global pour une approche du paysage fondée sur les nouvelles technologies de l'information et de la communication (NTIC) : élaboration progressive et analyse critique.

\section{5}

DEMOULIN Alain : Contribution à l'étude néotectonique de l'Ardenne-Eifel et des régions avoisinantes.

GOB Frédéric: La lichénométrie appliquée à l'étude de rivières en gorge en milieu méditerranéen : caractérisation de leurs paramètres dynamiques et de leur évolution géomorphologique durant le Petit Age Glaciaire.

HALLEUX Jean-Marie: Structuration spatiale des marchés fonciers et production de l'urbanisation morphologique: application à la Belgique et à ses nouveaux espaces résidentiels.

HOUBRECHTS Geoffrey: Utilisation des macroscories et des microscories en dynamique fluviale : application aux rivières du massif ardennais (Belgique).

\section{6}

CORNELIS Bernard: Towards a spatial decision theory.

DEWITTE Olivier : Cinématique de glissements de terrain et prédiction de leur réactivation : approche probabiliste dans la région d'Oudenaarde. 
LAPLANCHE François : Environnement de conception de bases de données spatiales sur Internet.

\section{- Université Libre de Bruxelles} Iles Desertas. Une contribution à la volcanostratigraphie et à l'évolution de l'archipel de Madère et à l'échelle de polarité du champ géomagnétique.

63

\section{6}

64 GIS applications for the development of Coastal/Marine Environment.

\section{Mémoires de licence - Licentieverhandelingen}

\section{- Katholieke Universiteit Leuven}

66 AERTS Koen : Erosie- en colluviatiegeschiedenis van de archeologische site van Tienen Grijpen. 

bewoners. Jelsi (Molise). Gelrode.

\section{5} Ecuador.

BOGMAN Pieter : Bijdrage tot het opmaken van een sedimentbudget op lange termijn (Holoceen) voor het Nethenbekken.

BOURGEOIS Stephanie : Bijdrage tot de geomorfologische opbouw van landduinen : het duinencomplex te Hechtel.

DOSSCHE Koen : Accumulatiegeschiedenis van het beken van Bereket. Bijdrage tot de reconstructie van het paleomilieu op het territorium van Sagalassos (Zuidwest-Turkije).

GEYSEN Lien : Onderzoek naar de invloed van publieke instellingen op de residentiële segregatie in Curitiba, Brazilië.

MEEUS Bruno: Een geografie van attitudes tegenover sociale huurwoningen. De Aarschotse wijk ‘Gijmelberg' als case study.

MEUWISSEN Ellen : Ruimte en socialisatie bij schoolgaande jongeren in Herk-de-Stad.

TRESIGNIE Sarah: Wijkontwikkeling in het Brussels Hoofdstedelijk Gewest: wijkcontracten en gentricificatie in de Brabantwijk te Schaarbeek.

Truyen Joeri : Ruimtelijke evolutie van enkele foehnepisodes over de Alpen.

ULENS Inge : Geografie van woonwagenparken en de maatschappelijke integratie van de

VAN BELLE Lise : Brusselse thuislozen : een geografie van woontrajecten.

VAN HEMELRIJCK Hendrik : Erosiesnelheden en landgebruik voor kleine stroombekkens in de centrale Ecuadoriaanse Andes.

VANDERLINDEN Lore: Ruimtegebruik bij Turkse vrouwen in Diest: wijzigingen ten gevolge van het huwelijk.

VANDERMOSTEN Gert : Toerisme ontwikkeling in een staatsgeleide economie : toepassing van ontwikkelingsmodellen op het Cubaanse toerisme.

VANDERSTRAETEN Lieve : Buitenlandse migraties van Italië in de $20^{\text {ste }}$ eeuw. Casestudy :

VANDEWALLE Annelies : Landgebruiksddynamiek in de hoge Ecuadoriaanse Andes : het bekken van de Burgay.

2 VERSTAPPEN Hannelore : Evolutie van het buitenhuis eten in de tweede helft van de $20^{\text {ste }}$ eeuw in gemeenten met een verschillende verstedelijkingsgraad.

WEVERS Wim : Evaluatie van maatregelen tegen water- en modderoverlast : gevalstudie

AERTS Klaas : Residentiële differentiatie en overlevingseconomie in Senegal : een casestudy in de voorstad Pikine (Dakar).

BATS Mia : Optimalisatie van het landgeberuik in een stroombekken met historische mijnactiviteiten : Recsk (Hongarije).

HANSSENS Katrien : Residentiële differentiatie en overlevingseconomie in Senegal : een case study in de voorstad Pikine (Dakar).

HERREMANS Sofie: Evaluatie van de infiltratiekarakteristieken van zones met verschillend bodemgebruik met behulp van regenvalsimulatie in het bekken van Jadan, 
LESAGE Stien : Invloed van de aanwezigheid van een grote stad op het bodemgebruik in de omliggende rurale gebieden in de Zuid-Ecuadoriaanse Andes.

MARTENS Veerle : Ruimtelijke en temporele spreiding van instortingen in ondergrondse kalksteengroeve in Zuid-Limburg.

POELMANS Lien : Invloed van de landgebruiksveranderingen op de sedimentfluxen in et Pécsely bekken, Hongarije.

OTTEVAERE Wim: Belgische multinationale ondernemingen : een typering op basis van directe buitenlandse ondernemingen.

SCHUERMANS Nick: Een geografische analyse van armoede bij blanken in Bloemfontein, Zuid-Afrika.

SMETS Toon: Sedimentological and hydrological changes induced by vegetation in gully areas.

VANDEN BROUCKE Lien : Economische impact van het voetbalbedrijf Manchester United op stad en regio : een dynamische factor?

VAN DE SYPE Piet: Impacts of small-scale mining on erosion and sediment field in small catchments in the Northern Ethiopian Highlands.

VANHULLE Kim : Sedimenttransport in de bovenloop van het Gibabekken, Ethiopië.

VANWEVERBERG Kwinten: Stedelijk warmte-eiland Brussel. Een modelmatige schatting van het aandeel stedelijke warmte in de temperatuurreeks te Ukkel.

VERDUYN Rubia: Indicatoren ter voorspelling van sedimentexport op stroomgebiedschaal in Spanje.

\section{6}

BEEL Annelies: Assessment of sediment export in the upper part of the Geba catchment, Ethiopia.

DEVOS Evelyne : Endo- en exogene actoren bij de economische reconversie van Nord-Pasde-Calais.

GHEYSEN Simon: Transport opportunities and food shopping behaviour in a context of development: case: Cape Town.

KINNAER Tinne : Evaluatie van het plattelandsbeleid met focus op de problematiek van de woon- en leefomgeving. Case-study : Hageland en Haspengouw.

LAURIJSSEN Tom : Invloed van de omgeving op de perceptie van de eigen gezondheid. Case studies in Limburg.

MEYS Sarah: People-place relationships at the neighbourhood level. Case study in a South African post-apartheid township.

PAUWELS Jan: Invloed van landgebruiksveranderingen op de sedimentfluxen in het Rimov bekken, Tsjechië.

S SCHERMANS Johan: Fluviatiele dynamiek van de Nijl te Deir-Al-Barsha (Midden-Egypte) : een verkennende studie.

SCHOUKENS Dieter : Sociale groepen en migraties in de deelgemeente Dilbeek.

VAN BOECKHOUT Sara: Actoren in hun interactie met ruimte bij jachthavenontwikkeling. Gevalstudie : Loosdrechtse Plassen (NL). 
VAN DEN BROECK Floris: Modelleren van landgebruiksveranderingen in Bohemen (Tsjechië).

VAN DEN PUTTE An: Experimental study of the buffering role of vegetated gully thalwegs. VAN GEIT Sarah: Samenhang tussen de naoorlogse dynamiek van Zuid-Pajottenlandse dorpen en de evolutie van hun buurtwegen.

VAN LERBEIRGHE Veerle: Plattelandstoerisme als motor voor regionale ontwikkeling: bijdrage door institutionele actoren (case: Uniso-projecten in de Westhoek en het Hageland).

VERBEEK Katrien: Braakliggende landbouwgrond in Vlaanderen: de band met verstedelijkingsdruk en natuurgebieden.

VERBRUGGEN Rozemarijn: De geologische metafoor toegepast op de residentiële ruimte in Lier : de Industriële Revolutie tot nu. Een actorenbenadering.

\section{- Rijksuniversiteit Gent}

\section{4}

\section{optie geografie}

ABBEEL Annick: Multitemporele analyse aan de hand van satellietbeelden van de landschapsevolutie in het Dachsteingebied, Oostenrijk.

AMEZ Tom : Van Coronabeeld tot DEM en morfografische kaart. Kartering van een gebied in het Altaïgebergte.

BAERT Erika : De Westhoek in het wereldsysteem : een verkennend onderzoek.

BONNY Krista: Geomorfologische studie van de Nijlvallei in de omgeving van AsSibaiyyah (Quena/Aswan, Boven Egypte). Een bijdrage tot het geo-archeologisch onderzoek van de pre-dynastische site El-Adaïma.

BOSSYNS Bert : Aanmaak van een DEM en een vegetatieclassificatie van een deel van het Salonga nationale park in de «Republique Démocratique du Congo » aan de hand van satellietbeelden.

BRADT Filip : Concepten van temporeel GIS toegepast op tweehonderd jaar kadastrale kaart.

DE BREUKER Dieter : De incorporatie van het Incarijk in het wereldsysteem.

DE MEYST Eva: De betekenis van de cultuurhistorische relicten in de BRU'NA BOINNE (Boyne-Vallei, Ierland) in de actuele landschappelijke context en dynamiek.

DE RICK Johan : Complexiteit en Geografie : een literatuurstudie.

DE SPIEGELEIRE Debbie : Mexico City in de wereldeconomie : meer dan een megastad in de periferie? Een beschrijvende studie van de stedelijke transformatie van Mexico City vanaf 1940.

DE WILDE Frederik: Studie van de landschappelijke ontwikkeling van de Wondelgemse Meersen te Gent a.d.h.v. eenkaartreeksanalyse van 1775 tot vandaag.

DIET Tristan : Bijdrage tot de geomorfologie van de Mont Canigou (Pyreneeën).

GOVAERT Bernard: Urbanisatie van het platteland, meer bepaald studie van de landschappelijke veranderingen en dorpszichten in de gemeente Maldegem (van Ferraris tot heden). 

Europa.
HUYZENTRUYT Wim : Rivierbekkenbeheer : Vergelijkende studie tussen het Netebekken en het Ourthebekken.

KESBEKE Wouter : De kwestie Irak : Een botsing tussen beschavingen.

LAMBERT Lars : Bijdrage tot de geomorfologie van het Land van Waas.

LOONES Jordy : Rekeningrijden een mogelijke garantie voor vlot stadsverkeer?

SCHELFAUT Kristien : Studie van het ozongat boven Antarctica.

SCHELFAUT Anke: Geomorfologische en geo-archeologische studie van de "Valle de Ricote " (Riio Segura, Region de Murcia, Spanje).

SPITAELS An: Vergelijkende studie van het Vlaams minderhedenbeleid in de OostVlaamse steden Aalst, Lokeren, Ronse, Sint-Niklaas enTemse.

TERRYN Griet : Paleo-ecologische geschiedenis van Lago Puyehue (Chileen Merendistrict) gedurende de laatste 17.000 jaar: een reconstructie aan de hand van siliceuze microfossielen.

VAN BOGAERT Rik (Erasmus): Snow avalanche hazard in Pyhätunturi tourist zone, Lapland province, Finland.

VAN DEN BRANDE Marianne : De Hedendaagse Wereldorde bekeken vanuit Realistisch en Marxistisch/Globalistisch perspectief. Een vergelijkende literatuurstudie met toepassing op de kwestie Irak.

VAN DER GUCHT Wannes : De rol, kwaliteit en beeldvorming van kaarten in kranten. Een analyse aan de hand van voorbeelden uit de Irakoorlog (2003).

VAN HOVE Katrien: Historisch en kritisch onderzoek naar de gegevens van de topografische kaart van België 1/20.000 van Philippe Vander Maelen.

VAN HOYWEGHEN Jana : Verbossing en bosbegrazing als methoden voor landschapszorg in Vlaanderen. Evaluatie van een aantal lopende projecten.

VAN MARCKE Wouter : Territoriale organisatie van Joegoslavië en de opvolgerstaten.

VAN MEERBEECK Cédric: De Golfstroom en zijn relatieve weerslag op het klimaat in

VANSTEENKISTE Vincent: Ruimtelijke ontwikkeling en sociale differentiatie van de Brugse agglomeratie vanaf 1830.

VERBEKE Bart: Onderzoek naar verplaatsingsvoorkeuren in Gent aan de hand van «Stated Preference » Technieken.

VERMAEL Matthias : Marokko in het kapitalistisch wereldsysteem.

VERRYT Soetkin : Studie van het fysisch milieu van de « Région de Murcia » (Spanje).

VERSTRAETE Lies : Een bijdrage tot de studie van de geomorfologie en de geo-archeologie in de omgeving van het antieke Potentia (Porto Recanati, Provincia di Macerata, Regione delle Marche, Italia).

WERBROUCK Lore: Typologie en evolutie van de percelering op het schiereiland The Hook (Country Wexford, Ierland) aan de hand van luchtfoto interpretatie en terreinstudie.

optie landmeetkunde 
154 ANDRIES Paskal (samen met Christophe FASSEUR) : Onderzoek naar de mogelijkheden van de fotogrammetrische restitutie van oppervlakken met een uitgesproken relief toegepst op OLV-Kathedraal in Doornik.

BRUNEEL Lieselot: EGNOS, het Europees augmenttiesysteem voor satellietnavigatie en GALILEO, het toekosmtig Europees satellietnavigatiesysteem: systeembeschrijving, architectuur, status en onderzoek naar EGNOS ontvangst in stedelijk en open gebied.

DANCET Pieter : Evaluatie van DEM's verkregen uit terrestrische fotogrammetrie aan de hand van terreinmeting (toegepast op de abdij van Aulne, Thuin).

DENISSEN Marjolein : Landschappelijke inpassing van infrastructuurwerken.

FASSEUR Christophe (samen met Paskal ANDRIES) : Onderzoek naar de mogelijkheden van de fotogrammetrische restitutie van oppervlakken met een uitgesproken relief toegepst op OLV-Kathedraal in Doornik.

GOEMAN Kathleen (samen met Loes HEYRMAN): Topografische analyse van clothoïdevormen in de geometrische wegenbouw.

HEYRMAN Loes (samen met Kathleen GOEMAN): Topografische analyse van clothoïdevormen in de geometrische wegenbouw.

TACK Frederik: Het opstellen van een mogelijke methodologie voor het gebruik van terrestrische fotogrammetrie ten behoeve van archeologische opgravingen.

VAN BELLEGHEM Didier (samen met Bram VAN MEULEBROECK): Geometrische en veiligheidsaspecten van verhoogde inrichtingen op de openbare weg: verkeersdrempels en verkeersplateaus.

VAN MEULEBROECK Bram (samen met Didier VAN BELLEGHEM): Geometrische en veiligheidsaspecten van verhoogde inrichtingen op de openbare weg: verkeersdrempels en verkeersplateaus.

VERBEECK Pieter: Akoestische en sedimentologische karakterisatie van een oude munitiedumpplaats : de Paardenmarkt site, Belgisch continentaal plat.

\section{Socrates}

KORKMAZ CERYAN Nurchihan: Potential Instability Zonation of Natural Slop on Harsit Granitoid using GIS.

SANZ PENA Berta: Landscape analysis of the waterways and greenveins design in the city of Ghent: the case of the Bourgoyen-Ossemeersen.

\section{5}

\section{optie geografie}

BASSENS David: Een bijdrage tot de studie van de geomorfologie en de geoarcheologie van het dal van de Rio Segura stroomopwaarts van Ciena (Région de Murria, Spanje). Het westelijk deel.

BLOMMAERT Natasha : Ruimtelijke aspecten van leefbaarheid in de Vlaamse Ardennen: vergelijkend onderzoek Oudenaarde en Horebeke.

CAPPON Roeland: Ruimtelijke analyse van de geselecteerde open-ruimteverbindingen in de sterk verstedelijkte Leieband in Zuid-West-Vlaanderen. Doorkijk naar ontwikkeling en planning van open-ruimteverbindingen.

CLOSSE Jürgen: Ruimtelijke dynamiek van de sierteelt in de kleine kernen ten noordoosten van Gent. 
COOLS Ann : Inventarisatie en historisch-kritische studie van de cartografie van het Land van Waas van de $18^{\text {de }}$ en $19^{\text {de }}$ eeuw.

DECRAEMER Marloes : Evaluatie van Asterdata voor de aanmaak van een hoogtemodel en een bodembedekkingsclassificatie (toegepast op Zuid-België).

DE SMET Maarten: Ontwikkelingen in de Open Ruimte. Een ruimtelijk-economische analyse met aandacht voor de voedingscluster in het Meetjesland.

DOBBELS Matthias : Windhozen in België.

DOSSCHE Rebekka (Erasmus stud.): Estudio geomorfológico y geoarqueológico del escarpe entre Alfocea y Juslibol, NW de Zaragoza, Aragón, Espana (Geomorfologische en geoarcheologische studie van de steilwnd tussen Alfocea en Juslibol, NW van Zaragoza, Aragón, Spanje).

HUYS Raphaël : Invloed van de Yzerinundaties op de Eerste Wereldoorlog.

KNOPS Caspar: Heide: een cultuurlandschap als natuur beheerd. De landschapsontwikkeling van het Grenspark De Zoom - Kalmthoutse Heide.

LABARQUE Minke: Paleolimnologische reconstructie van de Holocene omgevingsgeschiedenis van Pourquoi-Pas-Island (Antarctisch Schiereiland).

LAGAE Frédérique : Laat Pleistocene klimaatsreconstructie op basis van een lacustriene sedimentboring in Zuid-Chili.

LAPEIRRE Frederik : Een landschapsecologische situering en analyse van de " De Merode » bossen.

NIEUWBORG Dieter: Evaluatie van de efficiëntie van klassenindeling en legendevoorstelling op choropletenkaarten.

PIESSENS Wouter: Tropische Cyclonen: kenmerken, socio-economische impact en verband met global warming.

SAVAT Alanda: Onderzoek naar de mogelijkheden om schuine luchtopnamen te gebruiken in een 3-D kartering voor archeologische doeleinden.

THYS Barbara : Tokyo in de wereld-systeemanalyse.

TRISTE Laure : Een bijdrage tot de studie van de geomorfologie en de archeologie in de omgeving van het antieke Helvia Recina (Villa Potenza, Provincia di Macerata, Regione della. Westelijk deel.

VANDECAVEYE Niek : Profilering en ruimtelijke dynamiek van Lichtervelde in de regio Centraal-West-Vlaanderen.

VANDEVORST Wim: De geografische aspecten van de organisatie van de Olympische spelen in Barcelona '92 en Sydney 2000, met een doorkijk naar België en Vlaanderen.

VAN VOOREN Nick: De verbreding van het Schipdonkkanaal : ruimtelijke analyse, met accent op landschappelijke, sociale en economische aspecten.

VERMEIRE Irjen : Een bijdrage tot de studie van de geomorfologie en de geoarcheologie van het dal van de Río Segura stroomopwaarts van Cieza (Región de Murcia, Spanje). Oostelijk deel.

VERVAET Stephanie : De recente ontwikkelingen in het gebied van de Grote Meren in Afrika vanuit wereld-systeem perspectief.

VERVALLE Sanne : De ruimtelijke dynamiek van Kortrijk op de omgeving. 

Europa. kaart. natuurbeheer.

WEYHAEGHE Jasmijn : De invloed van de klei van Ieper op het front van WO-I in de sector Passendale-Mesen.

\section{optie landmeetkunde}

BRADT Filip : Concepten van temporeel GIS toegepast op twee eeuwen grensevolutie in

DE CUBBER Jos: Vergelijkende analyse van terrestrische 3D-laserscanning en fotogrammetrie.

DEMEY Daan: Beïnvloedende maatregelen omtrent de woon- werkverplaatsingen in Vlaanderen bij toenemende mobiliteit.

DE SERANNO Sarah : Een bijdrage tot de studie van de geomorfologie en geoarcheologie in de omgeving van het antieke Helvia Recina (Villa Potenza, Provincia di Macerata, Regione delle Marche, Italia). Oostelijk deel.

DEVRIENDT Lomme : Het meten van relaties tussen wereldsteden. Zoektocht naar een zo goed mogelijke dataset.

GEUSENS Leen: Ontwikkeling van een beslissingsmatrix ten behoeve van het uitvoeren van terrestrische GRB-bijhoudingsmetingen.

JACQUES Sven : Een economisch-geografische studie met doorkijk ruimtelijke economie en planning van de circuits van Zolder vs. Francorchamps.

KNOCKAERT Alysse : Driedimensionele visualisatie en historisch ruimtelijke evolutie van een site aan de kanaalzone bij de Dampoort te Gent.

MARIËN Liesbeth : Driedimensionele visualisatie van een site aan de Dampoort te Gent in vergelijking met andere 3D-datasets.

MEEUS Bart: Analyse van de meetnauwkeurigheid van totaalstations o.m. aan de hand van interferometrie.

MORTIER Liselotte: Multi-scalaire cartografie ten behoeve van Geo-Archeologisch onderzoek - Gevalstudie Khishâm (Syrië).

NEUTENS Tijs : Onderzoek naar de (in)homogeniteit van de transformatiezones in het Flepos-netwerk (transformatie tussen ETRS 89 en Lambert 72 - coördinaten).

OTTEVAERE Wim: De historische ontwikkeling van de grenzen van de D.R. Congo in

PEIREN Idris : Netwerkanalyse op het openbaar vervoersnet van Gent in functie van een draagkrachtonderzoek.

SCHAUBROECK Wouter : Vergelijking van verschillende mapserving produten, toegepast op de visualisatie en analysemogelijkheden van tijdruimtelijke gegevens voor

SCHELDEMAN Rinus: Methodiek van landmeetkundige opmetingen: uitwerking van enkele case-studies.

3 VAN BEVER Benjamin: De aanmaak van een DEM, orthofotoplan en hoogtelijnenkaart voor archeologisch gebruik, aan de hand van Corona KH-4B beelden (Toegepast op de Yustidvallei, Rusland, Altaï Republiek).

4 VAN DEN BOSSCHE Koen: 3D-visualisatie van een geo-archeologische opgraving te Carthago (Tunesië). 
WALLAEYS Lars: Bespreking topografisch instrumentarium vanuit historisch en functioneel perspectief.

WATERINCKX Katrien: Constructieve en vormgevende maatregelen om bij toenemende mobiliteit de verkeersveiligheid te verbeteren.

2006

\section{optie geografie}

ADRIAEN Fien : Malaria in beweging : een strijd tussen mens en natuur.

AELVOET Els : Een bijdrage tot de studie van de geomorfologie en geoarcheologie van het dal van de Río Segura in de omgeving van Almadenes (Región de Murcia, Spanje). Westelijk deel.

BEKAERT Tim : Bepalen van de accuraatheid van ruimtelijke metingen op stereobeelden en sequenties van stereobeelden zoals gebruikt in mobile mapping systemen.

BOSCH Samuel: De invloed van het weer op de landschapsbeleving. Studie aan de hand van enquêtes aan de Lierse vest.

BRUYLANT Derek : Geopolitieke oriëntering en economische transformatie in de postcommunistische landen van Europa en het GOS : een SEM-analyse.

CALJON Laetitia: Geomorfologische studie van het meest noordelijke deel van de Maginotlinie met bijzondere aandacht voor het fort van Bersillies en omgeving.

CLAESSENS Bart : Een stadsgeografische analyse van « gated communities ». Effectuering van een analytisch model in de Vlaamse context, regio Antwerpen.

COMMEENE Delphine : Fysisch milieu en ontwikkeling : het Akagerapark in Rwanda

DEBAVEYE Hannes : Digitale beeldprocessing van ASTER-data voor de aanmaak van een DEM en een bodembedekkingsclassificatie (toegepast op Zuidelijk Euboia (Griekenland).

DECROOCK Veronique: De optimale inrichting van een jachthaven, casus jachthaven Merelbeke.

DE FLOU Mathias : Studie van het fysisch milieu van het eiland Socotra (Jemen) : klimaat en geomorfologie.

DELAFONTAINE Matthias : De invloed van continue en discontinue veranderingen van een netwerk op QTC $\mathrm{N}_{\mathrm{N}}$-relaties.

DENORME Emma: Toepasbaarheid van de methode «Historic Landscape Characterisation » op het lokale niveau in Vlaanderen. Geval-studie in de gemeenten Kruishoutem, Oudenaarde en Zingem.

de RAYMOND Joëlle: Studie van beach-rocks in zuidelijk Attika (Griekenland) als zeespiegelindactoren.

DE WILDE Jan: De problematiek van de hoogbouw in België. Doorkijk naar de case Antwerpen.

DE WINTER Ruben : De geomorfologie van de Kalkense Meersen ingepast in het kader van de meandersys-temen van de Beneden-Schelde tussen Gent en Dendermonde.

D'HOKER Niklaas Analyse van de stadsgewestelijke en stedelijk migratie-stromen in Vlaanderen tussen 1993 en 2004.

FRANKL Amaury: Sporen van glaciale morfologie in het massief van de Canigou (oostelijke Pyreneeën) aan de hand van een detailstudie in de Coumeladevallei 
GERANIOS Virgini : Het ontstaan van tsunami's en hun gevolgen: de tsunami van 26 december 2004 in Zuidoost-Azië.

GESQUIERE Liesbeth : Relicten van de Britse militaire kaarten van 1918 rond Poperinge in het hedendaagse landschap.

GLORIEUS Bram : Studie van chlorofyl- en oppervlaktetemperatuur-patronen in de Rode Zee aan de hand van een tijdreeks MODIS-beelden.

HERZEEL Dries: Evaluatie van de bruikbaarheid van eCognition Professional bij het classificeren van satellietbeelden en kleuren-orthofoto's in combinatie met vectoriële grootschalige geografische informatie als grondwaarheid.

HEYVAERT Olivier : De ruimtelijke dynamiek van Aalst. De evolutie van een industriestad.

HOSTEN Pieter: Quartaire paleoklimaat-reconstructie aan de hand van isotopengeochemisch onderzoek op een stalagmiet uit de grot van Han-sur-Lesse, België. Een vergelijking van de methodologie tussen België en Vietnam.

KELLENS Wim: Het digitaal hoogtemodel Vlaanderen als basis voor hydrografisch onderzoek. Analyse van interpolatietechnieken en vergelijking met het NGI-hoogtemodel.

PHLIPPO An : Landschapsveranderingen sinds 1970 in het grensgebied Meetjesland

- Zeeuws-Vlaanderen aan de hand van een tijdsreeksanalyse.

ROMBAUT Heijke : Desertificatie in de « Región de Murcia » (ZO-Spanje).

ROSE Mario : Verkeersgeografische doorkijk naar de multimodaliteit in de Benelux :

concurrentie of samenwerking tussen het netwerk van hogesnelheids-treinen en lagekostenmaat-schappijen in de luchtvaart.

SALEMBIER Yves : De eruptie van Mount Pinatubo (Filippijnen, Luzón, 15 juni 1991) en de invloed ervan op klimaat en atmosfeer.

SCHOTTE Tom : Aanmaak van DEM's en bodembedekkingsclassificatie van ASTER en CORONA KH4B beelden voor archeologische toepassingen (toegepast op Titanië Griekenland).

SEGHERS Bart: Stedelijk rekeningrijden naar Londens model : een haalbaarheidsstudie voor Antwerpen en Brussel.

SIMOEN Reen : Een bijdrage tot de studie van de geomorfologie en geo-archeologie van het dal van de Rio Segura in de omgeving van Almadenes (Region de Murcia, Spanje). Oostelijk deel.

SINNAEVE Gudrun : Ruimtelijke analyse van toerisme en recreatie in de zee-strand-duinpolderovergang. Casestudie : De Panne en Koksijde.

TEMMERMAN Marijke : Desertificatie in de Hexi Corridor (Gansu Provincie, NoordwestChina).

VANDERGUCHT Lander: Moskou als mondiale stad? Studie aan de hand van Sassen's conceptualisatie.

VANDEVELDE Leen : Ruimtelijke analyse binnen de driehoek Blankenberge Thorntonbank - Zeebrugge : een combinatie van zee, haven, toerisme en polders.

VAN DE VELDE Lisa: Onderzoek naar de landschapsdynamiek in de Scheldevallei ten zuiden van Gent van de $18^{\text {de }}$ eeuw tot heden.

VAN DE VIJVER Evelyn : Ruimtelijke dynamiek van de N42 tussen Wetteren en Zottegem. 
VANHAECKE Nils : Het fysisch milieu van Bahrein : kenmerken, relatie met de mens en recente ontwikkelingen.

VAN LOOCKE Nele: Sociaal-culturele en esthetische waarden van het Leielandschap: voorbeelden uit Sint-Martens-Latem en Astene.

VAN OOST Lien : Platteland met toekomst in Kruishoutem.

VERBRUGGEN Raf : Een historisch-geografische studie van landschap en nederzettingen in het land van Zoersel-Wijnegem.

VERCRUYSSE Laurens: Millimetrisch onderzoek van varven : studie van de assemblage van Coccolieten en Diatomeeën van het quartaire Cariaco Bekken, Venezuela.

VERFAILIE Tim: Quartaire paleoklimaat-reconstructie aan de hand van isotopengeochemisch onderzoek op een stalagmiet uit de grot van Han-sur-Lesse, België. Met een vergelijking van de methodologie tussen België en Socotra.

VERMEULEN Jeroen: Implementatie en visualisatie van het triangulair tijdsmodel toegepast op de Belgische verkiezingsuitslagen.

VERVALLE Sanne : De ruimtelijke dynamiek van Kortrijk op de omgeving.

WERBROUCK Ilke : Recente evolutie van het Durmelandschap sinds het begin van de twintigste eeuw aan de hand van interviews, iconografie, kaarten en literatuur.

optie landmeetkunde

BEECKMAN Rebecca: Implementatie van de administratieve indelingen van het graafschap Vlaanderen in een GIS-omgeving (12 de eeuw - ca. 1800).

DEVENTER Loes : Bespreking van het land- en kaartboek van Ronse daterend uit 1684 en geometrische verwerking van de ruimtelijke informatie.

OOMS Kristien: Hoe 2-dimensionale GIS-analysetechnieken uitbreiden naar de derde dimensie? Algemeen overzicht en toepas-sing op punt in polygoontest.

RIP Kim : De opbouw van een fietsroute-netwerkmodel. Een casestudy i.s.m. Stad Gent.

SEGHERS Sarah: Evaluatie van snelheids- en debietmeetsystemen in het labo: theoretische en praktische aspecten.

VAN ASSCHE Bastiaan: Analyse en implementatie van ruimtelijke datastructuren en algoritmen ten behoeve van de visualisatie van bathymetrische datasets van punten.

VAN DE SYPE Elie: Een bijdrage tot de mestpollutieproblematiek in centraal WestVlaanderen aan de hand van een detailstudie in de gemeenten Beernem en Tielt.

VANGHELUWE Céline : De evolutie van het gebruik van de legendes in de gewestplannen en de ruimtelijke structuurplannen in de periode 1972-2006.

VAN HEYSBROECK Koen : De stedelijke problematiek in de $19^{\text {de }}$ eeuwse gordel : case studie Ledeberg.

VANOMMESLAEGHE Matthijs: Fotogrammetrische restitutie van Corona KH4B beelden voor de aanmaak van basisdocumenten voor archeologische survey (toegepast op de Dzjazator vallei-Altaï Republiek - Rusland).

VERHAERT Steven : Close range fotogrammetrische restitutie van de dam van Zeebrugge : Methodologische ontwikkeling van een karteermethode.

VERNIERS Katrijn: Cartotheekorganisatie: aspecten van metadata, catalografie en toegankelijkheid. 


\section{- Université catholique de Louvain} l'Espagne. Belgique. Hawaii.

2005 européen. siècle. télédétection.

COIPEL Ch. : Le comportement spatial du campagnol agreste Microtus agrestis face à la fragmentation de l'habitat.

CREPIN T. : Quantification des émissions de $\mathrm{N}_{2} \mathrm{O}$ des sols agricoles en Belgique en fonction du type du sol et du type de climat.

DABIN D. : Stocks de carbone organique des sols et utilisations des terres dans la région de La-Roche-en-Ardenne.

DE RUYDTS F.-X. : Modélisation du ruissellement en milieu semi-aride. Evaluation de la méthode du Curve Number Distribué. Application à la Sierra de Gádor, Sud-Est de

DERMINE A. : Etude comportementale : L'impact de la fragmentation sur les mouvements du campagnol roussâtre (Clethrionomys glareolus).

EVRARD O.: Evaluation de l'occupation saisonnière du sol, du remembrement et des mesures de conservation du sol sur les coulées boueuses dans le village de Velm.

GIJBELS P.-F. : Localisation optimale des milieux d'accueil de la petite enfance dans l'est du Brabant Wallon.

HERIN R. : Les déterminants du prix du foncier agricole. Analyse à l'échelle communale en

KERVYN de MEERENDRE M.: The Capabilities of Satellite Data to study the Morphology and Size of pyroclastic Constructs: Case Study of the Mauna Kea Cinder Cone Field,

NAGY M. : Les zones franches d'exportation.

SMET S. : Impact des inondations au Mozambique sur les écosystèmes et les communautés locales : analyse par télédétection et SIG.

VANDY F.: Modélisation de la culture des céréales en 2050 en Pologne sur base de variables économiques, climatiques et technologiques et la place du secteur primaire polonais dans l'Union Européenne.

WILLIAMS F. : Localisation optimale des bureaux de police en Wallonie.

BOURGUIGNON G. : Etude de la qualité de la couverture GSM dans le train sur la ligne 130A de la SNCB (Charleroi-Erquelinnes).

CORNETTE G. : Les compagnies low-cost, stratégies et incidences sur le transport public

FOSTY Ch. : Impact de la privatisation des terres sur la distribution spatiale de l'habitat aux alentours du Masai Mara, Kenya.

7 GALMART A.: Modélisation des changements climatiques en Arctique au cours du $21^{\mathrm{e}}$

GOYENS C. : Quantification et motifs des changements d'occupation du sol sur les terres consacrées à l'agriculture mécanisée dans le district de Narok (Kenya). Application de la 

Preto do Oeste.

2006 Espagne). Wallon.

2004

HAYNES J.: Analyse de la configuration spatiale du paysage et des processus de déforestation en Amazonie brésilienne. Les cas de Sao Félix do Xingú, Uruará et Ouro

LEJEUNE M. : Etude du ruissellement en milieu semi-aride. Caractérisation des surfaces et estimation du ruissellement.

LINARD C.: Rôle des routes dans la déforestation en Amazonie brésilienne : analyse statistique multivariée.

NERI P.: Variation spatiale du prix de vente des maisons en Wallonie.

QUEECKERS S. : Localisation optimale des bureaux de poste dans le Brabant Wallon.

STINGLHAMBER Q.: Etude d'un pays en transition. Divergence régionale du développement de la Chine.

VANDENBULCKE G. : Localisations optimales et distributions de la demande dans l'espace. Simulations sur réseaux théoriques.

AREMOU S.: Changement de l'utilisation, de l'occupation du sol et fragmentation du paysage dans le district de Nkhotakota, région centrale du Malawi (1986-2001).

BELLIN N. : Impacts de la modification du parcellaire sur la connectivité hydrologique et l'érosion hydrique d'un bassin versant situé dans un milieu semi-aride (région de Murcie,

CHALANTON I.: Impacts des inondations causées par l'augmentation du niveau moyen des mers sur les activités humaines présentes à la Côte belge.

DOMBRET N.: Analyse de la sensibilité et d'incertitude sur un modèle d'expansion urbaine européen.

ESPALARD E.: Localisation optimale des services publics d'incendie dans le Brabant

FALLY J. : Les Fonds structurels européens : moteur de convergence régionale?

FONTEYN A. : Répartition spatiale du tourisme en Cornouailles (Royaume-Uni).

FRANCART I.: Impact de l'urbanisation de Louvain-la-Neuve sur les populations de micromammifères rongeurs dans les îlots boisés.

LAMARQUE P.: Facteurs associés à la distribution spatiale de la transmission de la Borréliose de Lyme en Belgique. Analyse statistique multivariée.

Le POLAIN de WAROUX Y.: Understanding Livelihood Choices around the Maasai-Mara National Reserve, Kenya. A statistical analysis using bivariate probit regression.

ROGALA A.: Regional convergence. Evaluation of standard of living throughout regions of the European Union.

\section{- Université de Liège}

\section{option géographie fondamentale}

BASTIN Aurore: Espaces verts et qualité de vie en milieu urbain: application à la commune de Liège. 
BODART Catherine : Etude de l'ensablement dans la région de Gouré (sud-est du Niger) : processus et apport de la télédétection.

COLOT Fanny: "Santé et territoire »: analyse des inégalités sociales dans l'arrondissement et la commune de Liège.

COOLS Sébastien : Le Goldberg et la dispersion de sa cendre en direction de la Belgique.

CORNEZ Valérie: Le développement des éoliennes en Wallonie: analyse critique des facteurs de localisation.

DEROCHE Florence : Etude paléoécologique de détail aux alentours de la transition BoréalAtlantique dans les lithalses de la fagne des Deux-Séries (Hautes-Fagnes, Belgique).

DOGUET Anne : Les sites charbonniers wallons : bilan des réaffectations.

FREIRE COIMBRA Jorge: Les migrants portugais entre le village d'accueil et le village d'origine : étude de géographie sociale dans les villages de Larochette (L) et de Fiolhoso (P).

HEINEN Nicolas : Mobilité et réseaux sociaux dans la Commune de Hamoir.

HEUSCHEN Benjamin: Etude téphrostratigraphique de dépôts holocènes du lac Icalma (Chili).

HULS Jérémy: Etude géo-archéologique du «champ mégalithique de Wéris »: la zone d'Oppagne, Wéris et Morville.

JENNES Emilie : Systèmes d'orientation dans une petite ville : application aux villes de Spa et Malmédy.

JOUNIAUX Marie : Evolution du littoral entre Gênes - Voltri et Arenzano en particulier les plages des environs de Vesima.

LUQUE Juan-Carlos : Gestion d'un territoire en vue d'un développement durable : étude de cas dans le Rif central (Maroc septentrional).

NICOLAY Estelle : Genèse et développement d'un axe commercial : cas concret de la route du Condroz.

SCHEPERS Jean-François : Les haies dans le réseau écologique de la commune de SommeLeuze : évolution, diagnostic et proposition d'optimisation.

SNIJDERS Jean-Philippe : Délimitation des espaces de liberté des rivières à des échelles temporelles emboîtées : application aux rivières ardennaises.

TAXHET Muriel : Etude des variations du littoral à l'ouest de Naples (Italie) à l'aide de données archéologiques.

VAN CAMPENHOUT Jean: Analyse des inondations en région liégeoise sur base des relevés des services d'intervention.

VAN DURME Gabrielle: Variabilité spatio-temporelle de l'albédo: analyse menée aux résolutions métrique et kilométrique.

WINKIN Lionel: Analyse de la logique de localisation et des pratiques du tennis en Belgique.

option géométrologie

CHAIDRON Christophe: Modèle conceptuel de base de données toponymiques associées aux objets du Seamless Geographic Information System of Reference (I.G.N.). 
COLLIGNON Anne-Sophie: Catalogue de données des objets de fontainerie de la Compagnie Intercommunale Liégeoise des Eaux d'après la norme ISO 19110.

LEDENT Amélie : Applicatif SIG au service Patrimoine de la CILE.

PLOUVIER Bruno : Détermination des caractéristiques d'une caméra numérique aérienne.

SPITS Justine : Développement d'un logiciel pour la simulation de mesures de pseudodistances Galileo et validation sur base de données GPS.

2005

\section{option géographie fondamentale}

ANTOINE Sylvain : Projets de villes et développement régional : étude du cas de Charleroi.

AVENDAÑO MORENO Vanessa : Le secteur du transport logistique en province de Liège : essor récent et répartition spatiale.

BALTUS Ludovic: Etude des caractéristiques et de la localisation des sociétés de courrier express en Belgique.

BONMARIAGE Sylvain: La réaffectation des grandes fermes en Wallonie: analyse de quatre territoires en province de Liège.

BOVY Benoît : Détection des niveaux de stabilité fluviale à partir d'un modèle numérique de terrain : développement d'une méthode basé sur le cas de la Vesdre.

CHEN Di : Processus de développement et mutation des espaces en Chine : existe-t-il un modèle de Wenzhou?

COLLIGNON Arnaud: Exploitation du SIG et des encoches d'érosion pour la mesure du déficit en sédiments et de l'érosion latérale en relation avec les modifications d'occupation du sol en 200 ans : application au bassin de la Somme (Condroz - Famenne).

KEUTGEN Philippe : Les parcs logistiques en Europe du Nord-Ouest : développement et localisation.

KRIER Isabelle : Apport de la télédétection à l'étude de l'évolution de l'occupation du sol et de l'aménagement côtier dans la région de Haiphong (Vietnam).

LEVECQ Yannick : Etude dynamique des principaux affluents ardennais de la Semois.

PAULY Xavier: Les secondes résidences en province de Luxembourg: recherche non exhaustive des facteurs de répartition.

RIXHON Gilles : Contribution à l'étude géomorphologique du bassin du Ninglinspo.

ROUSSEL Stéphanie : Cartographie des risques naturels à Dinant.

SIMON Quentin : Influence de l'oscillation nord atlantique (ONA) sur les températures et précipitations mensuelles : analyse menée à partir des données de Uccle et de 10 stations de référence en France.

STASSE Guillaume : Etude de l'envasement du lac de Bütgenbach.

option géométrologie

BLÜGE Thierry: Influence de l'effet multi-trajet sur les mesures de pseudo-distances effectuées grâce au système GPS.

DE BASTIANI Pietro : Schémas de classes articulés sur le concept d'unité cadastrale dans le cadre du projet CADGIS.

DETIFFE Michaël : Implantation d'une ligne TGV fret à l'aéroport de Liège-Bierset. 
FOURNEAUX Delphine: Portail interopérable d'informations géographiques: méthodologie et prototypage.

FRAIKIN François : Etude des coûts de viabilisation des parcs d'activités économiques en province de Liège.

HALLOT Pierre: Généralisation conceptuelle par modèle: application aux données du $1 / 50$ 000e de l'IGN.

LATINIS Virginie : Automates cellulaires : application à l'évolution périurbaine de Liège.

LEROY Benoit: Proposition de cartographie de la région du Bas-Congo en République démocratique du Congo.

MAYERES Nicolas : Impacts géomorphologiques d'un ouvrage d'art sur un cours d'eau : le cas de l'autoroute A26 sur la Chavanne.

MODAVE Loïc : Structure a posteriori du réseau de voiries contenu dans le PICC.

PILONETTO Jonathan : Evaluation du modèle de correction troposphérique appliquée au système de positionnement EGNOS.

POUMAY Nicolas: Validation de la modélisation du levé par laserométrie du pont de Franière appliquée à un élément de structure.

ROUSSEAU Etienne: Méthodes de détermination d'obstacles autour d'un aérodrome: application à l'aérodrome de Spa - la Sauvenière.

SERVAIS Xavier : Etude du nouveau système de référence «Lambert 2005 » et analyse de son impact.

SPITAELS Frédérique : Problématique de la généralisation de la carte topographique au $1: 20$ 000e de l'I.G.N. : proposition d'une solution compatible avec le modèle «SGISR ».

TARGE Jean-Christophe : La future plate-forme intermodale de Hermalle-sous-Argenteau : implantation de voies de communication.

TIHON Geoffrey: Développement d'une méthode de validation structurale d'un MNS affecté d'une erreur altimétrique: application au MNS généré à partir d'un couple d'images SPOT5-HRS sur la région liégeoise.

VON HOFFMANN Nikolaï : Validation d'un modèle numérique de surface par stratification des données : MNS extrait d'un couple d'images SPOT5-HRS de la région liégeoise.

option géographie fondamentale

DETALLE François: Evaluation de risques que représente l'ancienne activité charbonnière. Le cas du terril du Hasard à Retinne.

DION Elise : Etude diachronique de la dynamique des sables et de l'impact d'un projet de lutte contre l'ensablement (projet «Ceinture Verte») à Nouakchott (Mauritanie) grâce aux apports de la télédétection.

DURY Marie : Contribution à l'étude du rayonnement solaire à la station météorologique du Mont Rigi - Transparence de l'atmosphère et albédo.

GUILLAUME Nicolas: Etude de l'influence de la centrale hydroélectrique de Heid de Goreux sur la morphologie de l'Amblève et sur l'utilisation de l'habitat physique par des poissons rhéophiles entre Nonceveux et Sougné-Remouchamps. 

verviétoise. Italie.

HILIGSMANN Stéphane : La réaffectation des friches économiques. Le cas de la région

KEUTIENS Sébastien : La collecte sélective des déchets par parc à conteneurs - Etude des disparités spatiales dans la zone Intradel.

KEVERS Virginie : La localisation des usines de biodiesel en Allemagne, en France et en

LABIE Raphaël: Inventaire et analyse des mécanismes impliqués dans l'évolution de l'agriculture de la commune de Chièvres.

LEMOINE Pierre: Evolutions du secteur agricole en Nouvelle-Zélande. Quels impacts régionaux suite au passage à une agriculture non subsidiée?

LEROY Thibaut : Etude de paramètres physico-chimiques et hydrologiques de sources. La région d'Erezée, Mormont et Wéris.

LEZY Thibault : Contribution des tomographies électriques et des sondages par sismique réfraction à la géographie physique.

MARISCHAL Sophie : Les rénovations du cadre bâti dans la Corbeille namuroise : mise en évidence des facteurs d'influence et analyse de leur diffusion spatiale sur base des dossiers des mutations du cadastre.

MERCIER Grégory: Spécialisation, concentration et mixité fonctionnelle dans les quartiers en difficulté. Le cas de Verviers.

PAULY Alexandra: Analyse des hausses de température nocturne à la station météorologique de Raeren.

PIRON Julie: Contribution à l'étude du climat de trois cavités souterraines belges. La grotte de Ramioul, la grotte et abîme de Comblain-au-Pont et la carrière souterraine de Petit Lanaye inférieure.

ROTH Jean-François : Etude diachronique, à l'aide de la télédétection, de l'évolution de la plaine côtière de Chân Mây (Vietnam).

ROUCHET Hélène : La localisation des stations de car-sharing en Wallonie. Le cas de la clientèle particulière.

RUCHE Emilie : Etude d'un outil public de l'aménagement du territoire opérationnel : les zones d'initiatives privilégiées - quartier d'intervention (Z.I.P. - Q.I.) - Cas des quartiers Sainte-Marguerite et Saint-Léonard à Liège.

SMETS Marie : Analyse spatiotemporelle de la température de surface des eaux du lac Tanganyika. Mise en évidence de l'impact d'ENSO.

THEUNISSEN Yannick : Etude de l'évolution de l'état de surface de la chaussée en fonction des conditions climatiques. La période hivernale d'octobre à avril.

option géomatique - géométrologie

BAUDINET Gilles : Etude du relevé tridimensionnel d'une paroi rocheuse.

BONTEMPI Jérémie : Conception et réalisation de sites didactiques dédiés aux méthodes topographiques de base.

ERNST Julien: Modélisation tridimensionnelle de fragments urbains par voie photogrammétrique. 
GOUDERS Pierre: Amélioration de la méthode d'extraction des MNS obtenus par correlation d'images SPOT5-HRS: détection d'altitudes non conformes et technique d'interpolation.

WADELEUX Marc: Influence de la configuration géométrique de la recherche de points homologues sur un couple d'images SPOT-HRS par matching spectral.

WAUTELET Gilles : Etude des TID's à l'aide des GNSS : influence de la géométrie de la constellation.

\section{- Université libre de Bruxelles}

\section{4}

HANRIOT Grégoire: La pêche européenne: répartition et évolution des captures de poissons marins.

JACOB Denis: Apport des outils de développement territorial au tourisme dans deux communes du Luxembourg belge. Bertrix et Habay.

LEGRAND Julien: Etude de la biodiversité du peuplement de macro-invertébrés et caractérisation de la variabilité géographique de la qualité biologique des eaux dans le bassin de la rivière l'Argentine (La Hulpe).

LEROUX Vincent: Une analyse globale du système monde: étude quantitative et typologique.

LEWALLE Laurence : La variation spatiale des prix des loyers à Bruxelles.

LOUVIAU Julien: Utilisation de la composition isotopique du strontium des eaux de source pour la détermination de la contribution de l'altération au cycle du calcium en hêtraie ardennaise.

ROMAINVILLE Alice : Une « flamandisation » de Bruxelles? Analyse empirique.

TENZER Laurent : Géographie du changement politique au Mexique.

ZANATTA E. : La localisation du commerce de luxe à l'échelle mondiale.

\section{5}

ANDRE Raphaël : Evolution des structures économiques des régions d'Europe médiane.

BAUWELINCKX Marie: Les transformations du monde agricole sous l'effet de la périurbanisation en Brabant wallon.

DEFUISSEAUX Geoffrey : Apport de l'utilisation conjointe d'images optiques et radar pour la détection des indicateurs de présence ou d'absence de mines antipersonnelles : Etude de cas : le site de Blinjski Kut en Croatie.

De SCHOUTHEETE Cécile : Evolution récente de l'urbanisation résidentielle aux Pays-Bas et en Belgique. Rôle des pouvoirs publics.

DUJARDIN Juliette : Détermination de la composition isotopique du strontium des hêtres en Forêt Régionale de Soignes pour l'identification des sources d'alimentation en calcium.

GEILFUS Nicolas-Xavier: Etude expérimentale de l'équilibre des carbonates lors de la formation de la glace de banquise.

GERARD Marie : Conditions de coexistence des cultures génétiquement modifiées avec les cultures conventionnelles et biologiques dans la mosaïque du paysage wallon. 
GHILAIN Adeline : Comparaison de l'impact du petit et du grand commerce de détail sur les flux de circulation (autos et camions).

MASSON Florence : Signatures isotopiques de l'origine du fer et des processus biologiques liés au cycle biogéochimique du fer dans la zone côtière de la Mer du Nord.

MEZZOUR Saïd : La rénovation urbaine et la population locale dans un quartier à forte présence d'origine étrangère : « Le vieux Molenbeek ».

PRIOUX Renaud: La diffusion des énergies renouvelables. Deux échelles d'analyse: l'Union Européenne et la Belgique.

REMY Jean-Philippe : Caractérisation du réseau microbien de la glace de mer côtière du détroit de McMurdo (Antarctique).

SIMON Stephan: Etude de la qualité physico-chimique et biologique des eaux de ruissellement dans le bassin du Geleytsbeek (Uccle).

SUMBUL Lieve : Quels sont les facteurs influençant la répartition spatiale de la vacuité des étages au-dessus des commerces de détail à Bruxelles?

VANHOSMAEL Sahra: Répartition spatiale des étudiants des Hautes Ecoles bruxelloises comparée avec celle des étudiants de l'Université Libre de Bruxelles.

WAUTHIER Thibault : Evolution des localisations spatiales du bâti dans les zones du plan de secteur.

2006

ADILE Khalid : Géographie des lieux du culte musulman à Bruxelles.

BASTIN Gaston : La mobilité des personnes employées par les établissements de plus de 200 travailleurs en Région de Bruxelles-Capitale. Synthèse et analyse des plans de déplacements d'entreprise.

BAUDHUIN Stéphane : Charleroi, une ville sportive? Une approche géographique.

BAUDSON Mathieu : Impact des nouvelles vagues d'immigration sur les centres urbains au Canada : le cas des Chinois de Montréal.

CALUGARU A-S.: Epistémologie comparée de l'enseignement de la géographie en Roumanie et en Belgique depuis le XIX ${ }^{\mathrm{e}}$ siècle.

5 DE BROUWER Gilles: Le glacier rocheux de Mullins Valley dans les Dry Valleys en Antarctique préserve-t-il un signal paléoclimatique fiable ? Le cas d'un sondage prélevé à proximité de la ligne d'équilibre du glacier.

DEBROUX Tatiana : L'insertion socio-spatiale des artistes dans la ville. Le cas de Bruxelles.

DELCOURT Charlotte : Les glaciers de l'Alaska et le changement climatique.

DE MAEYER Mathieu: Comparaison internationale de la morphologie des quartiers de trois villes à partir d'images satellitaires à très haute résolution: les cas d'Abidjan, de Nairobi et de Karachi.

DESCAMPS Julien : Localisation, insertion dans l'environnement national et analyse socioéconomique et démographique des villes nouvelles en Europe occidentale.

d'HOOP Quentin: Implication dans les changements climatiques de la production de particules exopolymériques transparentes (TEP) chez le coccolithophore Emiliana huxleyi.

ERMANS Thomas : Géographie des lieux de sortie à Bruxelles. 
GABIAM Koessan: Insertion et contribution des communautés gays et lesbiennes aux changements urbains à Bruxelles.

GABRIEL Valérie: Modélisation de l'emploi par type de commerce par commune en Belgique.

GODEAU Vincent : Etude de l'évolution temporelle des compositions en gaz de la banquise annuelle printanière au large de la station Casey (Antarctique) : relations avec l'activité biologique.

HORVAT Alain : Une géographie du vélo utilitaire en Belgique. Analyse multiscalaire.

JADOUL Nicolas : Géographie de l'espace économique wallon en 1910.

KUMMERT Pierre: Le glacier rocheux de Mullins Valley dans les Dry Valleys en Antarctique préserve-t-il un signal paléoclimatique fiable? Le cas de sondages prélevés dans la zone d'accumulation et dans la zone d'ablation du glacier.

LABBE Gaëtan: Géographie - à l'échelle mondiale - de l'offre en matière de tourisme d'aventure.

LEVY Yaïr: Essai de modélisation spatiale des populations d'Ips typographus en phase épidémique.

ROSE Charles : La diffusion spatiale des arts martiaux en Belgique francophone.

STRALE Mathieu : Géographie et stratégie des alliances de compagnies aériennes.

YDE Martine : Analyse de l'offre touristique des autocaristes wallons.

\section{- Vrije Universiteit Brussel}

\section{4}

COX Raf : Opslag van organisch koolstof in de bodems van de Vlaamse Zandstreek.

MEERSMANS Jeroen : Opslag van organische koolstof in de Vlaamse zandleembodems.

OTTOY Nils : Morfologische studie van de Leie- en de Scheldevallei in het Kortrijkse.

PENNÉ Kristel: Kartering van ondoorlaatbare oppervlakten op basis van sub-pixel classificatietechnieken.

VAN CAUWELAERT Wouter : Studie van de Boven-Pleistocene afzettingen in de Scheldeen de Leievallei (kaartblad Kortrijk).

VAN HOUTTE Kathleen: Ruimtelijke studie van sociale uitsluiting in Brusselse wooncomplexen.

\section{5}

CLAUS Jan : Korrelvorm van Pleistocene afzettingen in de Noorderkempen.

COOLS Sarah: Stedelijkheid in Brussel: nieuwe economische dynamieken in zeven wijken.

DE HAUWERE Nathalie : Ontstaan en evolutie van de Laat-Holocene getijdengeul Testerep, in het gebied tussen Nieuwpoort en Oostende (Belgische kustvlakte).

DE ROECK Tim : Kartering van ondoorlaatbare oppervlakken op basis van Landsat ETM+ en IKONOS satellietgegevens.

HUYCK Pieter: Modelleren van de massabalans van gletsjers op basis van NCEP-NCAR reanalysisdata. 
476 VAN DOOREN Veerle : De herbestemming van het Militair Hospitaal (Antwerpen) : Op zoek naar duurzame ontwikkeling in de wijken Haringrode, Oud-Berchem en Zurenborg. 2006

BAETENS Julie : Geomorfologisch onderzoek van de sequentie te Kesselt (België) aan de hand van ADIA en een 2D KS-test.

BOONEN Kevin : De ruimtelijke impact van eventuele Brusselse Olympische Spelen.

COPPENS Laurent: Objectgeoriënteerde classificatie van urbane gebieden aan de hand van IKONOS gegevens.

DE LATTHAUWER Kim : Ruimtelijke ordening van de sportinfrastructuur in het Brussels Hoofdstedelijk Gewest.

DEVENS, Hanne: Onderzoek naar de mogelijkheid van waterfrontontwikkeling aan de kanaalzone tussen de Ninoofsepoort en het Saincteletteplein in Brussel.

HEUNINCKX Shary : Vergelijkende studie van de toepassing van de nieuwe stedelijkheid in de ruimtelijke structuurplannen en cultuurbeleidsplannen van Brussel, Antwerpen en Gent.

LAMBRECHT Seppe: Onderzoek naar de sociale geografie van de Poolse gemeenschap (pen) in Brussel.

MOORTGAT Wim: Migraties en stedelijkheid: een analyse van het Belgisch migratiepatroon en stedelijke ontwikkeling 1992-2004 met een detailonderzoek van het stadsgewest Brussel.

ROTTIERS Pieter: Vergelijkende studie van de stadsvernieuwing in Sint-Niklaas, Mechelen en Leuven.

7 VAN DE PUTTE Karina : Diepteverdeling van koolstofopname in de Belgische leembodems in functie van bodemgebruik en drainage. 